\title{
Resilience to stress in bipartite networks: Application to the Islamic State recruitment network
}

April 12, 2022

Selena Wang and Jared Edgerton

Author Note:

Selena Wang, Department of Psychology, The Ohio State University; Jared Edgerton, Stanford University

Correspondence concerning this article should be addressed to Selena Wang, Department of Psychology, The Ohio State University, OH 43210. Email: wang.10171@osu.edu.

Key words: Network resiliency | Network knockouts | Dynamic systems | Terrorist recruitment 
Networks are resilient to internal failures or external attacks. The resiliency is often beneficial, but there are scenarios where the collapse of a social system, network, or organization would benefit society, such as the dismantlement of terrorist, rebel, or organized crime groups. In this article, we develop a methodology to estimate the effect of knockouts and apply our method to the Islamic State recruitment network. Using our novel application, we demonstrate how coordinated attacks against recruiters might reduce the Islamic State's ability to mobilize new fighters. This analysis has direct implications for studies of network resilience and terrorist recruitment. 


\section{Introduction}

Networks are resilient in the face of errors, failures, and external attacks (Albert, Jeong and Barabási, 2000b; Cohen et al., 2001; Gallos et al., 2005; Schneider et al., 2011; Gao, Barzel and Barabási, 2016; Eom, 2018; Laishram et al., 2018; Zhang et al., 2019). The resilience is based on network structures that can be found in various social, ecological, and technological networks, including the World-Wide-Web (Cohen et al., 2000; Barnett and Jiang, 2016), power grid systems (Chen and Hero, 2014; Dong et al., 2018), the economy (Griffith and Chun, 2015), human health (Bastiampillai, Allison and Chan, 2013; Kong et al., 2015), cell networks (Wuchty, 2014; Zitnik et al., 2019), ecological networks (Janssen et al., 2006; Baggio et al., 2016; Donohue et al., 2016), and social networks (Newman and Dale, 2005; Phan and Airoldi, 2015; Fernández-Martínez et al., 2017), among others (Yoo and Yeo, 2016; Scheffer et al., 2018; Zhang et al., 2019). Although local failures regularly occur within a network, they rarely lead to a collapse of networked systems with high connectivity, a small world structure, or scale-free degree distributions (Schneider et al., 2011; Gao, Barzel and Barabási, 2016; Eom, 2018). These features likely allow networked systems to localize and absorb the negative effects of failures brought on by internal errors or exogenous attacks.

The durability of networks helps prevent dire consequences, such as the widespread extinction of animals in ecological networks (Janssen et al., 2006), paralysis of power grid systems (Liu et al., 2016), or a pandemic from affecting entire populations (May, 2006). Yet, there are many circumstances when the collapse of a social system would benefit society, including the disruption of terrorist, rebel, and organized crime groups. Previous research has found that terrorist organizations are similarly resilient against exogenous shocks (Carley, Reminga and Kamneva, 2003; Carley, 2006; Moon and Carley, 2007; Price, 2012; Mannes, 2008; Milton and Price, 2020; Ryckman, 2020). This is due in part to these groups' network structure. For example, well-established terrorist and rebel organizations often have overlapping and redundant roles (e.g., leaders, facilitators, and recruiters) and local cliques and communities, or discrete and densely connected sub-groups of nodes (Matthew and Shambaugh, 2005; Malm, Nash and Moghadam, 2017; Ayling, 2009; Krebs, 2002). In turn, "decapitation strikes" against well-established terrorist and rebel organizations typically only marginally impact their lethality and longevity because the negative effects of an attack are confined and absorbed locally within the network (Jordan, 2009). 
However, networks are not always able to adapt to stress on the system, with research finding that networks are vulnerable to attacks targeting nodes central to the system's overall connectivity (Gallos et al., 2005; Broder et al., 2011; Albert, Jeong and Barabasi, 2000a; Jeong et al., 2001; Dunne, Williams and Martinez, 2002). This research suggests that terrorist recruitment may be vulnerable to strategic strikes if states correctly identify and remove members vital to the organization's connectivity. In this paper, we evaluate whether decapitation strikes can negatively impact networked terrorist organizations. In particular, we assess how strikes against communities (or cliques) of Islamic State recruiters affect the overall activity - i.e., the recruiters' ability to bring in foreign fighters.

The present article extends existing research in two unique ways. First, we study network resiliency using latent space models. And second, we apply this new methodology to understand terrorist recruitment - specifically, recruitment for the deadliest organization of the last decade, the Islamic State (LaFree and Dugan, 2007). Since its proposal (Hoff, Raftery and Handcock, 2002), latent space models have proven to be an appealing statistical model to account for dependencies in a network. Researchers have extended the latent space model to solve problems such as anomaly detection (Lee et al., 2019) and dynamic modeling (Friel et al., 2016; Sarkar and Moore, 2006; Sewell and Chen, 2015). A surge of new developments in this domain demonstrates its increasing use across areas of study (Salter-Townshend and McCormick, 2017; Handcock, Raftery and Tantrum, 2007; Friel et al., 2016; Sarkar, Siddiqi and Gordon, 2007; Minhas, Hoff and Ward, 2019; Dorff, Gallop and Minhas, 2020; Wang, Paul and De Boeck, 2021). Although latent space models have become increasingly popular, this approach has not been used to measure network resilience. This gap is notable given that standard probabilistic models focus solely on nodal degrees and ignore the rich relationships and dependencies among nodes that networks often exhibit.

We address this research gap by demonstrating how latent space models can be used to assess the resilience of networks in the face of targeted external attacks. Through this approach, we are able to capture the relational dependencies in a network and model the impact of targeted external attacks on network connectivity. ${ }^{1}$ This allows us to observe and precisely quantify a network's resilience at the nodal, clique, and system levels.

Compared with the nodal centrality-based analyses, such as the preferential attachment and

${ }^{1}$ Dependencies in a network are often labeled as popularity, sociability, transitivity, balance, clusterability, and stochastic equivalence (Hoff, 2008; Wasserman and Iacobucci, 1991). Here, we refer to network dependencies as the connectedness between nodes following their edges with each other. 
power-law models for networks (see Newman, 2003), the latent space based analyses possess a few advantages. Most notably, networks with similar nodal degree distributions are often assumed to be equally likely in the preferential attachment framework. Yet, these networks can have very different structures - e.g., different degrees of transitivity and community structures. In terrorist recruitment, there is a non-zero likelihood of community formation based on observed or latent attributes such as the demographics of the population, geography, shared language, and cultural heritage, among other commonalities shared between countries and geographic regions. Nodal centrality based analyses are unable to distinguish networks with different community structures when they exhibit similar nodal degree distributions. Therefore, the latent space based analyses can more accurately model the data generating process of the terrorist recruitment network.

In addition, the latent space visualizes how strongly a recruiter targets a country by calculating the Euclidean distance between them. Therefore, researchers and practitioners can study recruitment behaviors beyond nodes' centrality. Third, the latent space model is a flexible framework that allows us to assess the impact of knockout on an individual recruiter, the local clusters, and the network in general.

In addition to our methodological contributions, this article also contributes to extant research on terrorist and rebel mobilization. Previous research on how decapitation strikes affect terrorist organizations primarily rely on summary indices (Jordan, 2009; Johnston, 2012; Yaoren, 2019) — e.g., the size of or count of attacks carried out by the organization — or simulated networks to assess and analyze how these groups adapt to exogenous shocks (Carley, 2006; Moon and Carley, 2007). Our research marks the first time a real-world terrorist recruitment network data — with its full relational structures and complexities — is used to study combatant mobilization. Through this novel data, we are able to provide insights into the potential processes occurring within the organization following an attack. We find that the negative effect of external attacks is related to the lost activity of the knocked-out nodes and the network's resilience. Specifically, we find that decapitation strikes can potentially diminish the overall activity of the network and reduce the amount of mobilized foreign fighters. At the same time, we find that neighboring cliques and connected recruiters can be resilient to these attacks.

In the section below, we introduce the data and our modeling approach. This section is followed by an empirical assessment of the latent structures of the Islamic State recruitment network consisting of recruiters and their target countries and their cohesive local cliques (clusters). In the 


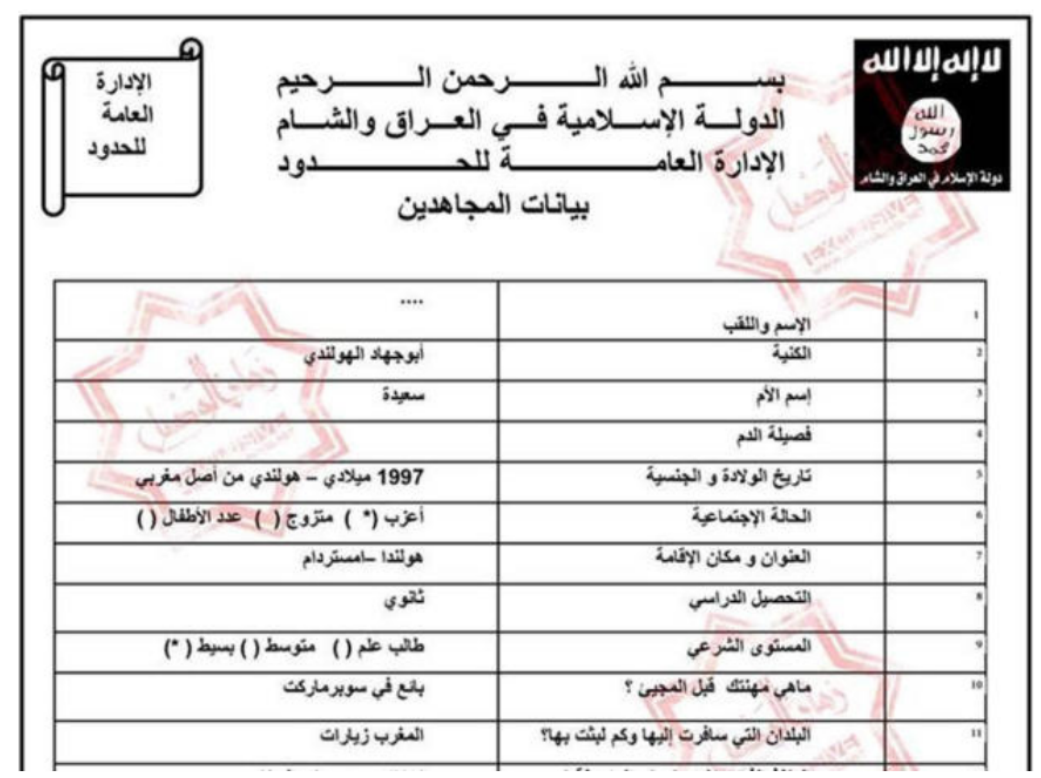

Figure 1: Example of Islamic State visa entry form from CBS News (CBS News, 2019). The visa entry forms had a wide range of questions, including who recommended the fighters to join the Islamic State and the fighters' home countries.

next section, we simulate targeted decapitation strikes on the recruitment network by eliminating pre-selected recruiters by cluster. We assess the effect of the knockout, or the extent of the network's resilience, by quantifying how the removal of the recruiters changes the network's overall activity level and neighboring groups' recruitment efforts. Last, we conclude.

\section{Materials}

After the Islamic State conquered parts of Iraq and Syria, they established themselves as a protostate, going as far as collecting taxes, acting as the judiciary, and performing other functions typical of governments (Callimachi, 2018). Among the records kept by the Islamic State were the visa entry forms of foreign fighters that had traveled to Iraq and Syria to fight for the Islamic State. These documents were given to our research team, as well as several media organizations and intelligence agencies that verified their authenticity (CBS News, 2019). These records include the fighter's home country and who recruited them to join the Islamic State (see Figure 1 for an example of the leaked visa entry forms). The research team cleaned the data in conjunction with their institutional review board (see the appendix for additional details on the data cleaning process). In addition to the present analysis, similar documents have been used by the World Bank (Abdel-Jelil et al., 2015) and West Point (Sterman, June 2018), among others (bin Khaled Al-Saud, 2019; Johnston et al., 
2016; Morris, 2020; Edgerton, 2022), for research purposes.

Across the visa entry forms, there are 1,276 unique individual recommenders. However, most "recommenders" were combatants' family members or friends. For the analysis, we define professional Islamic State recruiters as those who brought in more than ten combatants from 2013 to 2014 (see the appendix for a comparison between the recommenders' and professional recruiters' network). Unlike other prominent terrorist and rebel organizations, the Islamic State was able to recruit combatants through informal network ties (Hafez, 2016; Reynolds and Hafez, 2019), internet platforms (Chatfield, Reddick and Brajawidagda, 2015; Weimann, 2016; Speckhard et al., 2020), religious worship (Orozobekova, 2016; Nielsen, 2017) and even more formalized recruitment efforts, such as propaganda magazines (Bisgin, Arslan and Korkmaz, 2019; Lakomy, 2021). ${ }^{2}$ In total, there are 21 Islamic State recruiters in the data. These recruiters brought in 20 percent of the total combatants during that time from 36 states. In addition to bringing in a large volume of combatants, professional recruiters also brought in fighters from a more diverse subset of states, with a median of five countries per recruiter compared to a median of one country per recommender.

\subsection{Bipartite Recruitment Network}

We use the recruiter and state data to construct the Islamic State recruitment network as a bipartite network in the present analysis. In a bipartite network, edges are only present between nodes of different types. In the Islamic State recruitment network, the recruiters and states serve as two sets of nodes, and edges are only present between recruiters and states, with each edge signaling the count of combatants brought in from a country by a recruiter. Through the duality property of bipartite networks, information can be transferred between nodes. In the general literature, duality refers to the state of being two opposite but complementary properties. In network science, the definition of duality can be found in Wasserman and Faust (1994), and it refers to the duality of the relationship between actors and events. In the recruitment network, it refers to the complementary relationship between recruiters and countries. It refers to how recruiters are linked through their mutual involvement in countries. Likewise, countries are linked through recruiters. Connectivity between nodes of the same type is inferred through their shared ties to nodes of the other type.

In the Islamic State recruitment network, connectivity or association among the Islamic State

${ }^{2}$ In the Islamic State visa entry forms, combatants sometimes listed the names of known, or suspected Islamic State recruiters. Further, they would occasionally list a recruiter and how they corresponded with them - e.g., we spoke over the internet. 
recruiters is inferred through mutual involvement in the same countries. Figure 2 demonstrates this process. In Figure 2a-b, we see the one-mode networks for Islamic State countries and recruiters. Ties between two countries in cell (a) indicate whether the two countries are targeted by the same Islamic State recruiter, while ties between two Islamic State recruiters in cell (b) indicate whether the two recruiters brought in fighters from the same country. In cell (c), we present the bipartite network of countries and recruiters.

Figure 2d displays how connectivity and association are inferred within a two-mode network at the micro-level. In the example, recruiters $\mathrm{R}_{1}$ and $\mathrm{R}_{2}$ share ties to countries $\mathrm{C}_{i}$, while countries $\mathrm{C}_{1}$ and $\mathrm{C}_{2}$ share ties to recruiters $\mathrm{R}_{i}$. We infer that when recruiters target the same country, they are more similar ${ }^{3}$ than otherwise. Likewise, countries are more similar if the same Islamic State recruiters target them. In other words, if the same recruiter targets two countries, we say that these two countries share more in common than two countries that do not share the same recruiter. Thus, by claiming that countries are more similar if they are targeted by the same recruiter, we do not mean that countries are similar in geographic locations or demographics, or in any other colloquial sense. Instead, we mean that it signifies a tendency for statistical dependence between these two countries through a shared recruiter. We refer readers to Wasserman and Faust (1994) for a more detailed discussion on this topic.

Much like in a one-mode network, dependencies such as balance can be redefined in a two-mode network following its duality property. Structural balance, as a concept in network science, can be traced back to Heider (1946). Heider defines a balanced state between three nodes when all three possible ties are positive or when two are negative and one is positive. Mathematically, balance can be defined as when the product of three edges is positive (Hoff, 2005). Intuitively, balance simply suggests that friends share similar attitudes towards other people/entities and the lack of friendship (or negative edge) suggests divergent attitudes towards other people/entities. If neither of these holds, then there is no balance (Wasserman and Faust, 1994).

In this paper, we define recruiters' activity in different countries as a balanced state when the

${ }^{3}$ Note by "similar" we mean that there is dependence between countries when they are targeted by the same recruiter. For example, when two people like the same Instagram post, we say that these two people share a similar attitude about the same Instagram post. This indicates a statistical dependence (or positive correlation) between these two people if we look at many people's attitudes towards various Instagram posts. We can infer that these two people share more commonality with (or are more similar to) each other than two people who do not share the same attitude or two people who share different attitudes given that liking the same Instagram is not a random event or due to some other noise. 


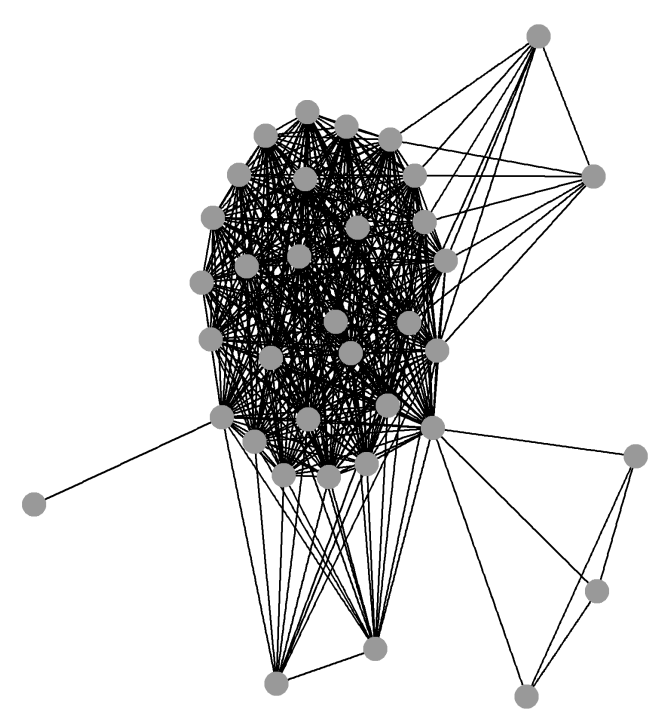

(a) One-mode country network.

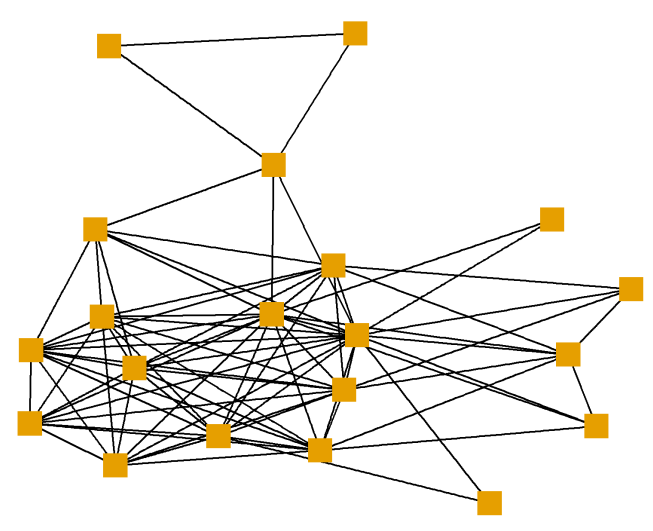

(b) One-mode recruiter network.

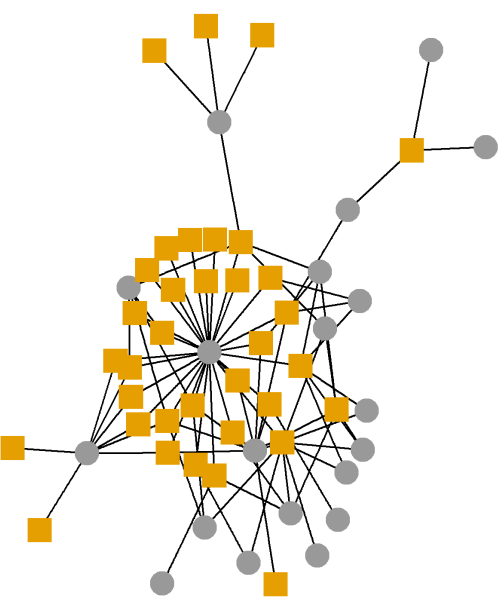

(c) Two-mode countryrecruiter network.

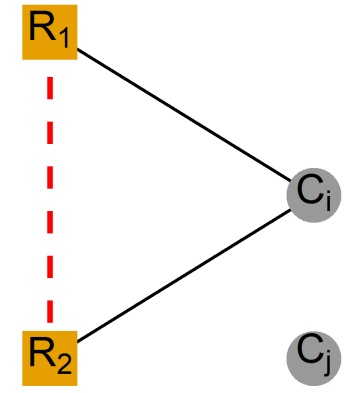

$\mathrm{C}_{\mathrm{j}}$

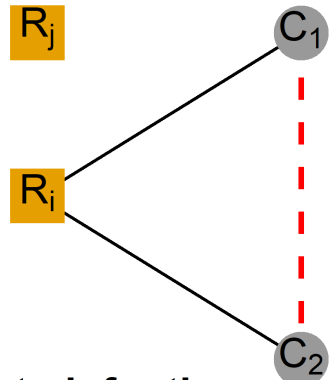

(d) Duality property to infer ties.

Figure 2: In cell (a), we can see the projected one-mode country network, with shared ties between states if recruiters brought in combatants from each state. In cell (b), we can see the projected one-mode recruiter network, with shared ties between recruiters if they brought in fighters from the same states. In cell (c), we see the bipartite network of recruiters and states, with recruiters sharing ties to the states they recruit from. Last, in cell (d), we see a demonstration of the projection, or how closeness is inferred by nodes of the same type in a bipartite network. The red lines indicate inference of closeness between nodes through their shared ties. 
product of three edges (two observed and one inferred) is positive. In a triad of one recruiter and two countries, balance occurs when a tie is inferred for two countries based on shared interest from one recruiter (see the dashed red line in Figure 2d); balance also occurs when a lack of tie is inferred for two countries based on differing interests from one recruiter. Different inferred ties have different implications for the observed ties. Intuitively, this suggests that a (latent) similarity or association can be inferred between recruiters when they target the same country, and a lack of similarity can be inferred when they target dissimilar countries.

\subsection{Network Patterns in Terrorist Recruitment}

In the present analysis, we employ a network approach to address the lack of independence between recruiter, country, and fighter activity. We model associations among recruiters and dependencies between counties as weighted ties, the number of foreign fighters brought in by a recruiter from a country. This approach is consistent with the existing research that emphasizes the importance of network structure in explaining the resilience and longevity of terrorist organizations (Memon et al., 2008; Lindelauf, Borm and Hamers, 2011; Medina, 2014). In this section, before discussing our analytic framework further, we situate the present research within the extant terrorism literature. Specifically, we discuss the implications of network dependence patterns - homophily, balance, and stochastic equivalence - in terrorist and rebel mobilization and conflict processes.

Researchers have noted that network homophily, or the tendency of nodes with similar attributes to share connections, can profoundly shape a host of political processes, including public opinion (e.g., Choi, Sang and Park, 2014; Bessi et al., 2016), ideology (e.g., Boutyline and Willer, 2017; Huber and Malhotra, 2017), political discourse (e.g., Weare, Musso and Jun, 2009; Colleoni, Rozza and Arvidsson, 2014), elite politics (e.g., Opper, Nee and Brehm, 2015), and protests (e.g., Centola, 2013; Dincelli, Hong and DePaula, 2016), among other issues (e.g., Maoz, 2012; Huber and Malhotra, 2017; Gallop and Minhas, 2021). Several scholars have applied these concepts to better understand rebel and terrorist mobilization (Magouirk, Atran and Sageman, 2008; Larson and Lewis, 2017, 2018; Larson, 2021). Larson and Lewis (2018) find that rebel groups use rumors to help consolidate their group during the early stages of rebellion. Specifically, they find that ethnically homogeneous peer and kin networks help rebel groups coordinate their efforts at the onset of a conflict.

Other scholars have discussed the importance of network balance. Network balance reflects dependence via shared values, attitudes, and opinions among friends. Put another way, when 
two people like each other, they tend to share the same evaluation (positive or negative) of other people, whereas when two people dislike each other, they tend to have different evaluations of other people (e.g., Heider, 1944, 1946; Norman et al., 1965; McPherson, Smith-Lovin and Cook, 2001). Several scholars have applied this concept to understand the connections between terrorist and rebel organizations (Asal, Ackerman and Rethemeyer, 2012; Horowitz and Potter, 2014; Bacon, 2018; Gade et al., 2019; Phillips, 2019). Asal et al. (2016) identified factors that lead to terrorist groups forming alliances. They found that organizations tend to ally with other groups that share their ideology (e.g., same ethnic or religious composition), are similar, and operate in the same countries.

Consistent with this scholarship, we expect homophily when recruitment occurs between entities with similar attributes - e.g., when recruiters target countries with which they share the cultural heritage. Following the definition of network balance in bipartite networks in section 2.1, we expect balance to occur in the terrorist recruitment network when a tie can be inferred between two countries $\left(C_{1}\right.$ and $C_{2}$, see Figure $\left.2 \mathrm{~d}\right)$ through shared interest from the same recruiter, $R_{i}$. When a tie is inferred between two countries $\left(C_{1}\right.$ and $\left.C_{2}\right)$, we expect them to have similar, both positive or negative (absent), relationships with other recruiters in the network. Similarly, we expect balance when a tie can be inferred between two recruiters $\left(R_{1}\right.$ and $\left.R_{2}\right)$ that target the same country, $C_{i}$.

In addition to homophily and balance, scholars have also explored the stochastic equivalence in political networks. Stochastic equivalence occurs when a groups of nodes in a network have similar relationships with other nodes. Dorff, Gallop and Minhas (2020) analyzed the rebel conflict network in Nigeria. They found that rebel groups in Nigeria follow a similar conflict pattern. Namely, although the rebel groups, Boko Haram, Movement for the Actualization of the Sovereign State of Biafra and Movement for the Emancipation of the Niger Delta came from geographically dispersed regions, they were similarly engaged in conflicts with the Nigerian government and police forces over territorial control.

This scholarship brings us to our second theoretical expectation. In the Islamic terrorist recruitment, we expect to observe stochastic equivalence, with groups of recruiters having similar recruitment behaviors, or targeting the same group of countries. 


\subsection{Resilience in Networks}

Resilience in networks refers to potential changes in networks when a node or a group of nodes are removed. A network is a representation of nodes' connectivity. When a node is removed from a network, its connecting edges disappear. In turn, the neighboring nodes will have to find a longer path to connect or lose their connections entirely. Networks vary in their resilience, and we can expect a network's resilience to depend on its topology and the properties of the lost nodes.

Scholars have generally found that randomly removing nodes has a nominal impact on the overall connectivity of the network. However, some scholars have found that targeting a specific class of nodes - e.g., high-degree nodes with many edges — may disrupt a networked system. Broder et al. (2011) found that even extreme attacks such as removing all nodes with degrees greater than five in networks such as the World-Wide Web still do not lead to the total collapse of the network. While this may seem like a drastic attack, in the real world, many networked systems are comprised of many nodes with few ties and a few nodes with many ties. Thus, it is often the case that in networks with highly skewed degree distributions, a large portion of the nodes have to be removed to cause a destruction of the network when the attack is random.

The impact of stress on networks depends on both the properties of the network and the properties of the removed nodes. Random attacks on exponential networks in which nodes have approximately the same number of edges are more effective than random attacks on scale-free networks in which nodes have a homogeneous number of edges (Albert, Jeong and Barabasi, 2000a). Targeted attacks on scale-free networks, where nodes with high nodal degrees are first targeted, are more effective than targeted attacks on exponential networks. For a scale-free network such as the Islamic recruitment network (see nodal degree distribution in Figure 3), we expect a larger impact of stress when more powerful (connected) recruiters are removed.

Given these considerations, we set out to measure the resilience of the Islamic State recruitment network in the face of targeted cluster attacks. Given the United States' key interest in the Islamic State and the close surveillance of the organization, we assume that attacks against the organization will have some knowledge of the networked organization's topology. We measure resilience as the changes in the network's connectivity following decapitation strikes (removal of recruiter nodes). This analytic approach is different from the previous research that identify nodes for removal based on nodal degrees because we identify recruitment communities for removal using the latent space 
framework. In the latent space framework, communities are identified by assuming that nodes' latent positions follow a mixture of Gaussian distributions and that each component of the mixture represents a different community (clique). The smaller the within-community variance, the more likely that a community emerges.

We focus on removing a community of recruiters for three reasons. First, removing a single node has not been found to change much of the network connectivity based on previous research, if at all. ${ }^{4}$ Second, it is easier to identify clusters of recruiters as they tend to bring in fighters from the same geographic regions. Indeed, previous scholarship found that recruitment activities can be localized to shared identities (Malet, 2013), communities (Larson and Lewis, 2018), neighborhoods (Hellsten, 2016; Reynolds and Hafez, 2019), and even sports teams (Atran, 2010, 2011). This scholarship suggests that recruitment efforts may be highly specialized such that rebels and terrorists are mobilized with combatants that share similar characteristics - e.g., speak the same language, have the same religion, or come from the same types of communities, among other commonalities. And third, the removal of recruiters has a direct policy implication - states often target terrorist and rebel recruiters to diminish the capacity of politically violent organizations and groups.

\section{Methods}

Our analysis is carried out in three parts. First, we model network dependency using the latent space framework and identify potential clusters. Second, we assess the resilience of the Islamic State recruitment network. We imitate knockouts of the recruiters and quantify how the knockouts affect the average recruiters' ability to mobilize fighters. Third, we demonstrate the influence of network dependencies on resilience using simulated data.

\subsection{Latent Space Model}

To identify clusters in the recruitment network, we first use latent space models to represent recruiters' activities in different countries in a hypothetical multidimensional latent space. The recruitment network is described by the $N \times M$ adjacency matrix $\boldsymbol{Y}$, where $N=21$ and $M=36$. The $(i, j)$ th element of the matrix, denoted as $y_{i j}$ is $c$ if recruiter $i$ recruited $c$ Islamic State fighters in country $j$ between 2013 and 2014 .

${ }^{4}$ In the appendix, we test if single node knockouts would similarly reduce recruitment. Consistent with previous scholarship, we find that removing a single node has a nominal effect on overall recruitment. 
While both Euclidean distances and vector products account for network dependencies, we use Euclidean distances because Euclidean distances are easier to interpret than the vector products. ${ }^{5}$ Let $\boldsymbol{U}$ be a $N \times D$ matrix of latent recruiter position, where each row is a $D$ dimensional vector $\boldsymbol{u}_{\boldsymbol{i}}=\left(u_{i 1}, u_{i 2}, \ldots, u_{i D}\right)$ indicating the latent position of recruiter $i$ in the Euclidean space. Let $\boldsymbol{V}$ be a $M \times D$ matrix of latent country position, where each row is a $D$ dimensional vector $\boldsymbol{v}_{\boldsymbol{j}}=\left(v_{j 1}, v_{j 2}, \ldots, v_{j D}\right)$ indicating the latent position of country $j$ in the Euclidean space. When the latent space model is applied to bipartite networks, the size of the Euclidean distance describes the intensity of connection between the two types of nodes, sometimes outside of the social friendship context (see Friel et al., 2016; Wang, 2021; O’Neale et al., 2019; Sarkar and Moore, 2005). When two nodes are closely connected, the Euclidean distance between their latent positions is small. A latent distance model for the bipartite Islamic State recruitment network can be written as:

$$
\begin{aligned}
y_{i j} \mid\left(\boldsymbol{U}, \boldsymbol{V}, \alpha_{0}\right) & \sim \operatorname{Poisson}\left(g\left(\phi_{i j}\right)\right), \\
g\left(\phi_{i j}\right) & =\exp \left(\alpha_{0}-\left|\boldsymbol{u}_{i}-\boldsymbol{v}_{j}\right|\right), i=1,2, \ldots, N, j=1,2, \ldots, M,
\end{aligned}
$$

where $\boldsymbol{u}_{\boldsymbol{i}} \stackrel{i i d}{\sim} N\left(0, \lambda_{0}^{2} \mathbf{I}_{D}\right), \boldsymbol{v}_{\boldsymbol{j}} \stackrel{\text { iid }}{\sim} N\left(0, \lambda_{1}^{2} \mathbf{I}_{D}\right)$, and $\alpha_{0}$ accounts for the density of the bipartite network.

We identify the cluster membership of recruiters and countries using the latent position cluster model (Krivitsky et al., 2009). To select the number of clusters, we evaluate the out-of-sample predictive performance under different numbers of clusters (see the SI Appendix for additional details). Through the clusters, we identify which recruiters target similar countries or coordinate their recruitment efforts.

\subsection{Network Resilience}

We simulate external stress to the recruitment network by performing a cluster knockout analysis. The effect of knocking out a cluster of recruiters is estimated as the average difference in the recruiters' propensity to recruit (PR) before and after the knockout. The knockout effect can be

\footnotetext{
${ }^{5}$ To interpret the latent space and the effect of interactions between latent vectors on the probability of an observed value under the vector product effect, we need to pay careful attention to the angle between latent vectors as well as their lengths, and in the case of additive and multiplicative effects model, the diagonal matrix between vectors. In contrast, we only need to pay attention to nodes' distances with each other using the latent distance model. The above reason makes the resulting latent space using the vector product more challenging to interpret, undermining one of the main strengths of the latent space approach. Therefore, we focus on the latent distance model, although the multiplicative effects model would be an interesting alternative.
} 
based on changes in the estimated intercept and latent positions (from the posterior distribution) or changes in the predictive new samples (from the posterior predictive distribution). The difference in $\mathrm{PR}$ is derived by re-estimating the intercept and the latent positions from the fitted latent space model after removing sets of recruiters. We define the difference in PR as the re-estimated PR values (after knockout) minus the original network's PR values (before knockout). Given the estimated latent recruiter and country positions, $\hat{\boldsymbol{u}}_{i}$ and $\hat{\boldsymbol{v}}_{j}$, we can estimate the PRs (based on the estimated parameters) before knockout as:

$$
g\left(\hat{\phi}_{i j}\right)=\exp \left(\hat{\alpha}_{0}-\left|\hat{\boldsymbol{u}}_{i}-\hat{\boldsymbol{v}}_{j}\right|\right)
$$

To estimate the knockout effect for a cluster, e.g. cluster A, we first re-estimate the PRs when the recruiters in cluster $\mathrm{A}$ are no longer in the model:

$$
g\left(\hat{\phi}_{i j}^{-A}\right)=\exp \left(\hat{\alpha}_{0}^{-A}-\left|\hat{\boldsymbol{u}}_{i}^{-A}-\hat{\boldsymbol{v}}_{j}^{-A}\right|\right)
$$

The latent position of recruiter $i$ changes following the knockout of (recruiters in) cluster A, from $\hat{\boldsymbol{u}}_{i}$ to $\hat{\boldsymbol{u}}_{i}^{-A}$. Then, the difference in PRs induced by removing cluster A based on changes in the estimated parameters is $g\left(\hat{\phi}_{i j}^{-A}\right)-g\left(\hat{\phi}_{i j}\right)$, and we define the knockout effect as the average of the differences, $\frac{1}{N M}\left(\sum_{i}^{N} \sum_{j}^{M} g\left(\hat{\phi}_{i j}^{-A}\right)-g\left(\hat{\phi}_{i j}\right)\right)$. To assess whether the magnitude of the difference between $g\left(\phi_{i j}^{-A}\right)$ and $g\left(\phi_{i j}\right)$ is large compared to the sampling variability of the data, we look at the posterior predictive distributions of new samples $\tilde{y}_{i j}^{-A}$ and $\tilde{y}_{i j}, \frac{1}{N M}\left(\sum_{i}^{N} \sum_{j}^{M} \tilde{y}_{i j}^{-A}-\tilde{y}_{i j}\right)$, given the observed values.

For the knockout analysis, we rerun the model with recruiters in cluster $A$ excluded and evaluate how the latent positions of the recruiters and countries change following the removal of cluster $A$. We then generate new predictions about their propensities to recruit based on their new latent positions. The predictions rely on new model parameters and the re-estimated latent positions of the recruiters and countries. Thus, the distances between the recruiters and countries change, and any difference we see in $g\left(\phi_{i j}^{-A}\right)$ from $g\left(\phi_{i j}\right)$ is a result of removing recruiters in cluster $A$.

More specifically, we expect that, when nodes are removed in a network, the remaining nodes will have to either find a longer path within the recruitment network to connect to foreign fighters, or face losing the connections entirely. Therefore, we expect increased distances between nodes 
following the knockout leading to decreased PRs. The size of the impact is determined by the differences in PRs between and after the knockout.

Recall that the impact of stress in networks is dependent on both the properties of the network and the properties of the removed nodes. Considering the scale-free property of the recruitment network, we expect targeted attacks based on nodal degrees to exert a negative impact on its overall connectivity. Considering that we target communities of recruiters identified using latent space models, we expect a higher knockout effect when the removed community (collectively among its recruiters) is more central to the networks' connectivity. Removing one Islamic recruiter is not expected to induce changes in the overall activity (see the SI Appendix for single recruiter knockouts).

We measure the network's resilience using the previously defined knockout effect. If a randomly selected recruiter engages less recruitment after the removal of a community of recruiters, we expect a substantial difference in the predictive new samples under the two models (before and after the knockout).

\subsection{Impact of Stress without Network Dependencies}

In this section, we assess the influence of dependencies on network resilience by devising a simulation study to compare knockout effects in networks with and without dependencies. We simulate data using the estimated intercept and latent positions following section 3.1. We compare the size of the knockout effects when there is dependence in the network versus when there is no dependence. Dependence in the network is generated by the nodes' Euclidean distances. The baseline networks are those with no dependence, and are generated as follows:

$$
\begin{aligned}
& g\left(\tilde{\phi}_{i j}\right)=\exp \left(\tilde{\alpha}_{0}\right), \\
& y_{i j}^{*} \mid \tilde{\alpha}_{0} \sim \operatorname{Poisson}(g(\tilde{\phi})),
\end{aligned}
$$

where $\tilde{\alpha}_{0}$ is the estimated posterior mean following section 3.1 , and $y_{i j}^{*}$ is the generated data. The comparison networks are those with dependence, and are generated as follows:

$$
\begin{aligned}
g\left(\phi_{i j}^{\prime}\right) & =\exp \left(\tilde{\alpha}_{0}-\left|\tilde{\boldsymbol{u}}_{i}-\tilde{\boldsymbol{v}}_{j}\right|\right), \\
y_{i j}^{\prime} \mid\left(\tilde{\boldsymbol{U}}, \tilde{\boldsymbol{V}}, \tilde{\alpha}_{0}\right) & \sim \operatorname{Poisson}\left(g\left(\phi^{\prime}\right)\right),
\end{aligned}
$$


where $\tilde{\boldsymbol{u}}_{\boldsymbol{i}}, \tilde{\boldsymbol{v}}_{\boldsymbol{j}}$, and $\tilde{\alpha}_{0}$ are the estimated posterior means following section 3.1. We compare the size of the knockout effects in the two types of data using the following procedures.

- For each dataset, we fit a bipartite Euclidean-distance latent space model and obtain the predicted edge values.

- For each dataset, we remove recruiters in the clusters identified in the latent position cluster model in section 3.1, fit a latent space model with the remaining data, and obtain the predicted edge values.

- Remove recruiters in cluster A, fit remaining data to the latent space model, and obtain the predicted edge values.

- Remove recruiters in clusters B,C, and D, respectively, fit remaining data to the latent space model, and obtain the predicted edge values.

- Compute the knockout effect for removing cluster A and clusters B, C, and D, respectively.

- Repeat the above steps 100 times and obtain two distributions of knockout effects for networks with and without dependencies.

If network resilience is related to network dependencies, then we expect smaller knockout effects in the dependent networks. More specifically, we expect networks generated with dependencies to be more resilient than networks generated without dependencies (independently generated observations) to cluster node knockouts. In other words, we expect the knockout effects to be larger in the baseline networks than the knockout effects in the comparison networks.

\section{Results}

\subsection{Latent Space}

Figure 3a displays the Islamic State recruitment network latent space in the 2-dimensional euclidean space. ${ }^{6}$ Clockwise from the nodes colored orange, we label the four clusters A, B, C, and D. Figure $3 \mathrm{~b}$ displays the recruiters' distances from the center of the Islamic State recruitment network latent space. Recruiters in clusters A and B are closest to the center, with a mean distance of 1.26 for cluster A, 2.02 for cluster B, 3.07 for cluster C, and a distance of 2.50 for cluster D.

${ }^{6}$ Two-dimensional euclidean spaces are commonly selected for easy interpretation and visualization. 

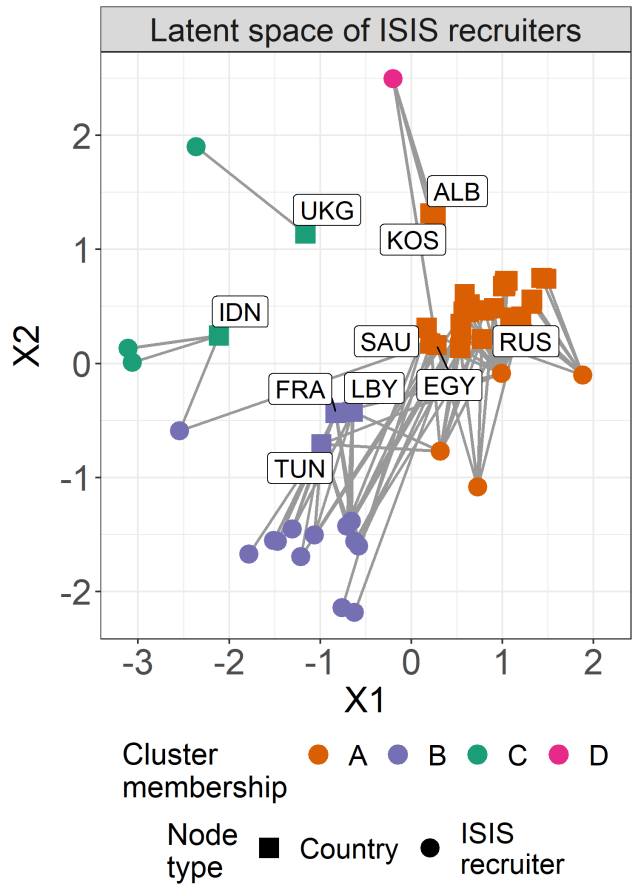

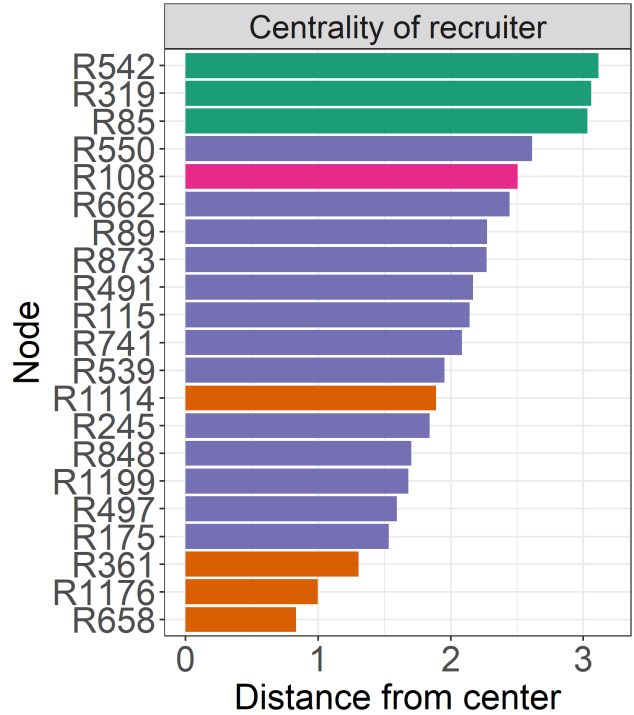

Cluster $\quad A \square B \square C \square D$ membership

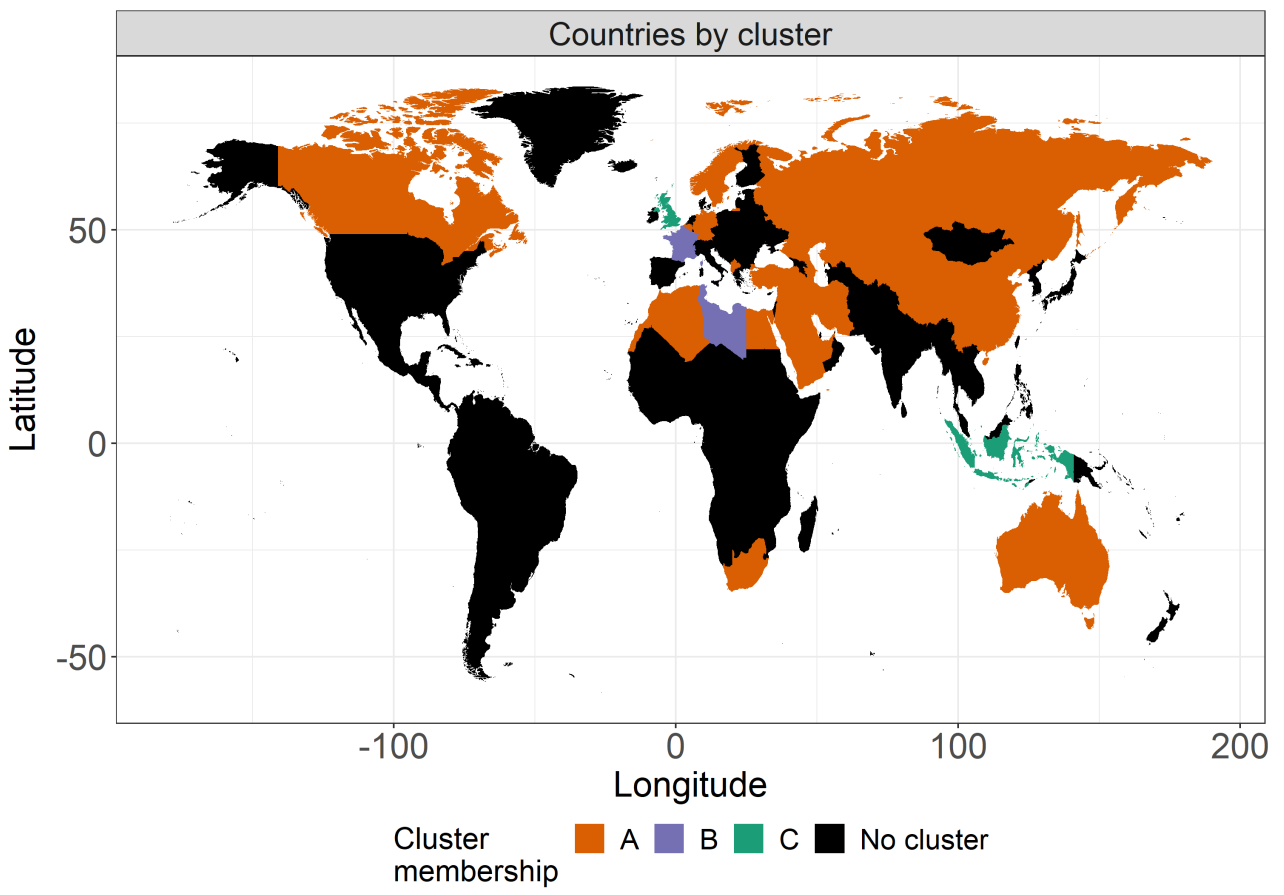

Figure 3: The latent space, centrality of Islamic State recruiters, and clusters membership of states. In cell (a), we see the bipartite latent space model for Islamic State recruiters and countries. In cell (b), we see which Islamic State recruiters by cluster membership as their Euclidean distance to the center of the latent space. Last, in cell (c), we see the cluster membership of all states. In addition to four recruiters, cluster A is composed of 31 states, including Albania, Algeria, Australia, Azerbaijan, Belgium, Bahrain, Canada, China, Egypt, Germany, Iran, Iraq, Jordan, Kazakhstan, Kosovo, Kuwait, Kyrgyzstan, Lebanon, Macedonia, Morocco, Norway, Russia, Saudi Arabia, South Africa, Sweden, Syria, Tajikistan, Turkey, the United Arab Emirates, Uzbekistan, and Yemen. In addition to 12 recruiters, cluster B is composed of France, Libya, and Tunisia. And, last, in addition to three recruiters, cluster $\mathrm{C}$ is composed of Indonesia and the United Kingdom. 


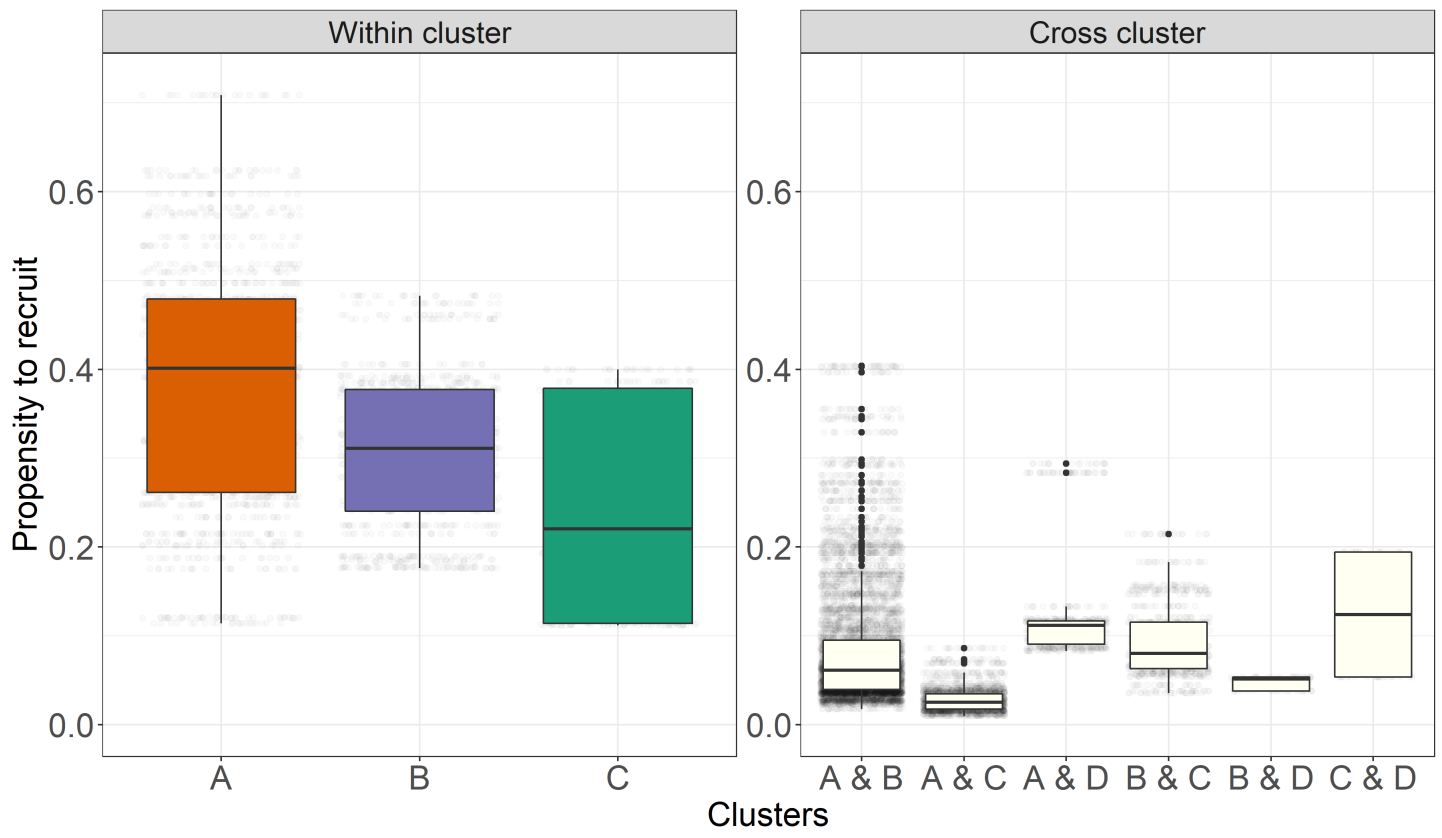

Figure 4: The propensity to recruit (PR) value for recruiters by cluster from the fitted latent space model. Recruiters in cluster A have the greatest PR, followed by B and C. Cluster D has no states within their cluster, so they only have inter-cluster recruitment activity.

Figure 3c displays the full states membership by cluster membership. In cluster A, four recruiters $(1176,1114,658$, and 361) are active in 31 countries across the Greater Middle East, Europe, and Asia, including Saudi Arabia, Jordan, Morocco, China, Russia, Turkey, Germany, among others. Recruiters 1176 and 1114 are the first and second most active recruiters, bringing in 118 and 53 foreign fighters. Together, cluster A recruiters bring in 213 foreign fighters, constituting 43 percent of the recruited foreign fighters. Cluster A has a low recruiter-to-country ratio and constitutes recruiters most central in the Islamic organization (see Figure $3 \mathrm{~b}$ ).

Compared to cluster A, cluster B has a high recruiter-to-country ratio, with recruiters engaging in more centralized and coordinated recruitment efforts. In cluster B, 13 recruiters heavily target France, Libya, and Tunisia. The clustering of France, Libya, and Tunisia in cluster B could be partially driven by these states' colonial histories, with France colonizing Tunisia in 1881 (Choate, 2007) and having military jurisdiction over southern Libya in the aftermath of World War II (Ahmida, 1994). Recruiters in cluster B are the second most central in the Islamic State recruitment network (see Figure 3b). In total, they bring in 226 foreign fighters, constituting 47 percent of the foreign fighters recruited.

Recruiters in clusters C and D have peripheral roles in the Islamic State recruitment network. In 

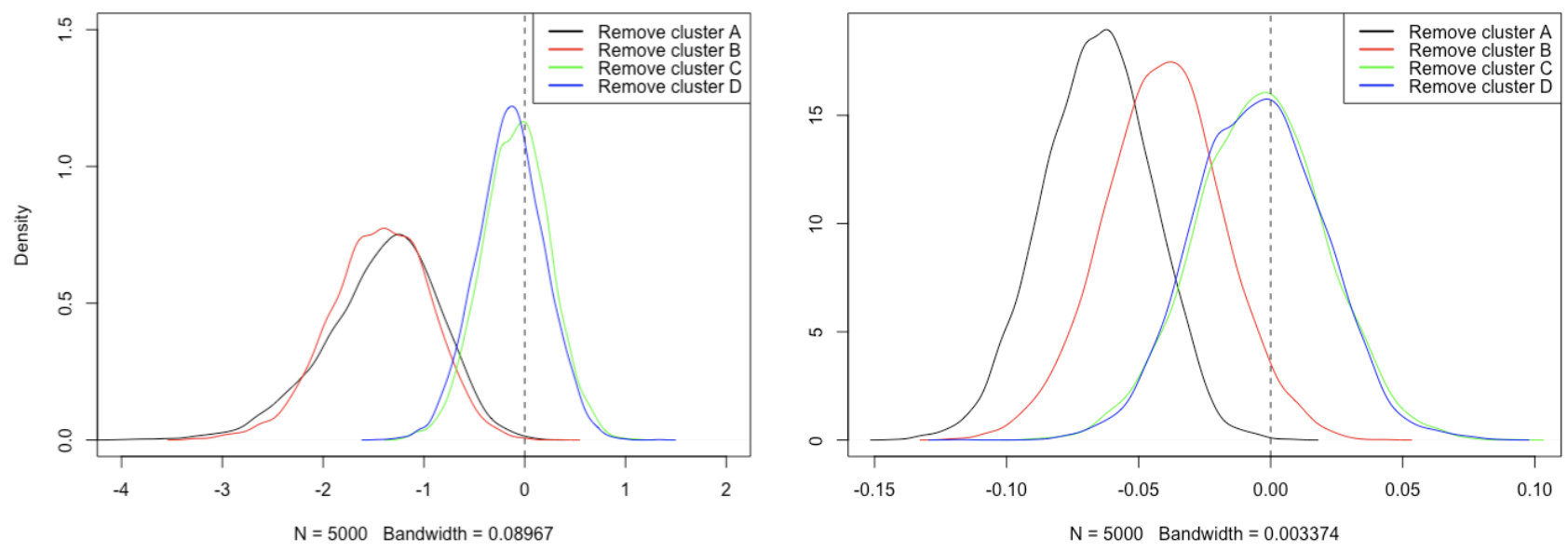

Figure 5: The knockout effects based on posterior means (left) and based on predictive new samples right) following simulated network knockouts. Knockouts of clusters A and B decrease the remaining recruiters' PR.

cluster C, the United Kingdom and Indonesia (again with historical colonial ties between the two) are targeted by three recruiters $(85,319,542)$, while cluster D only has recruiter 108. Recruiter 108 draws Islamic State fighters from three states in the Balkans (Kosovo, Albania, and Macedonia) and Egypt, all of which are countries in cluster A. Clusters C and D draw in fewer foreign fighters than clusters $\mathrm{A}$ and $\mathrm{B}$, bringing in a total of 35 and $11-7$ percent and 2 percent - of the total fighters.

Figure 4 helps us better understand recruitment activity by cluster by displaying the propensities to recruit (activity levels) within each and between clusters. Recruiters in cluster A have the highest internal recruitment activities, with a median PR of 0.40 , recruiters in cluster $\mathrm{B}$ have a median $\mathrm{PR}$ of 0.31, and recruiters in cluster $\mathrm{C}$ have a median $\mathrm{PR}$ of 0.22 . By comparison, inter-cluster connectivity is relatively low, with a median PR of 0.06 between clusters A and B, 0.03 between clusters A and C, 0.11 between clusters A and D, 0.08 between clusters B and C, 0.05 between clusters B and D, and 0.12 between clusters C and D. Since cluster D is comprised of a single recruiter, it has no intra-cluster recruitment activity, but relatively high rates of inter-cluster recruitment activity. 


\subsection{Network Resilience}

Figure 5 displays the changes in mobilization brought on by the simulated cluster knockouts. Notably, there is wide variation in the impact of the cluster knockout on the rate of mobilization for the remaining recruiters, with the knockouts of clusters A and B producing reductions in mobilization. Conversely, the knockouts of clusters C and D do not significantly reduce the rate of mobilization for the remaining recruiters, with both the differences in posterior mean and predicted value simulations crossing zero - i.e., suggesting no discernible effect on mobilization following the knockouts.

Figure 6 provides a cluster-level visualization of the knockout effect per knocked-out cluster, with the columns corresponding to the knocked-out cluster, the rows displaying the posterior mean and predicted value differences in recruitment. The boxplots display the changes in mobilization rates for the remaining recruiters by cluster membership. Points below the horizontal red line indicate a reduction in mobilized fighters compared to the pre-knockout rate of mobilization. Consistent with the Figure 5 results, Figure 6 shows that the knockout of clusters A and B reduces the estimated posterior mean and predicted values of foreign fighters across the remaining recruiters. In particular, the knockout of cluster A produces a noticeable decrease in the predicted number of foreign fighters mobilized by 0.03 (median) in cluster B and 0.05 in cluster D. In contrast, the observed reduction in mobilized fighters at the network level brought on by the knockout of cluster B is primarily caused by the removed recruiter $\mathrm{B}$.

Consistent with previous research, our results show that targeted attacks on scale-free networks can exert damage to the network. While previous literature looks at the impact on the overall network, e.g., the diameter of the connected graph, we are able to quantify the effects at the individual node level, of neighboring cliques, in addition to the effects on the overall network. Consistent with previous research, our results also show that targeting central nodes (based on nodal degrees) exert the most substantial damage. In our results, the removal of cluster A with the most active recruiters in the network exerts the strongest damage.

Different from previous literature, our results show that the damage following the simulated knockouts is not solely dependent on the number of nodes removed. While previous literature often shows an increased damage to the network following an increase in the number of nodes removed, our results show that this is not necessarily the case. Removing cluster B with a total of 13 recruiters 


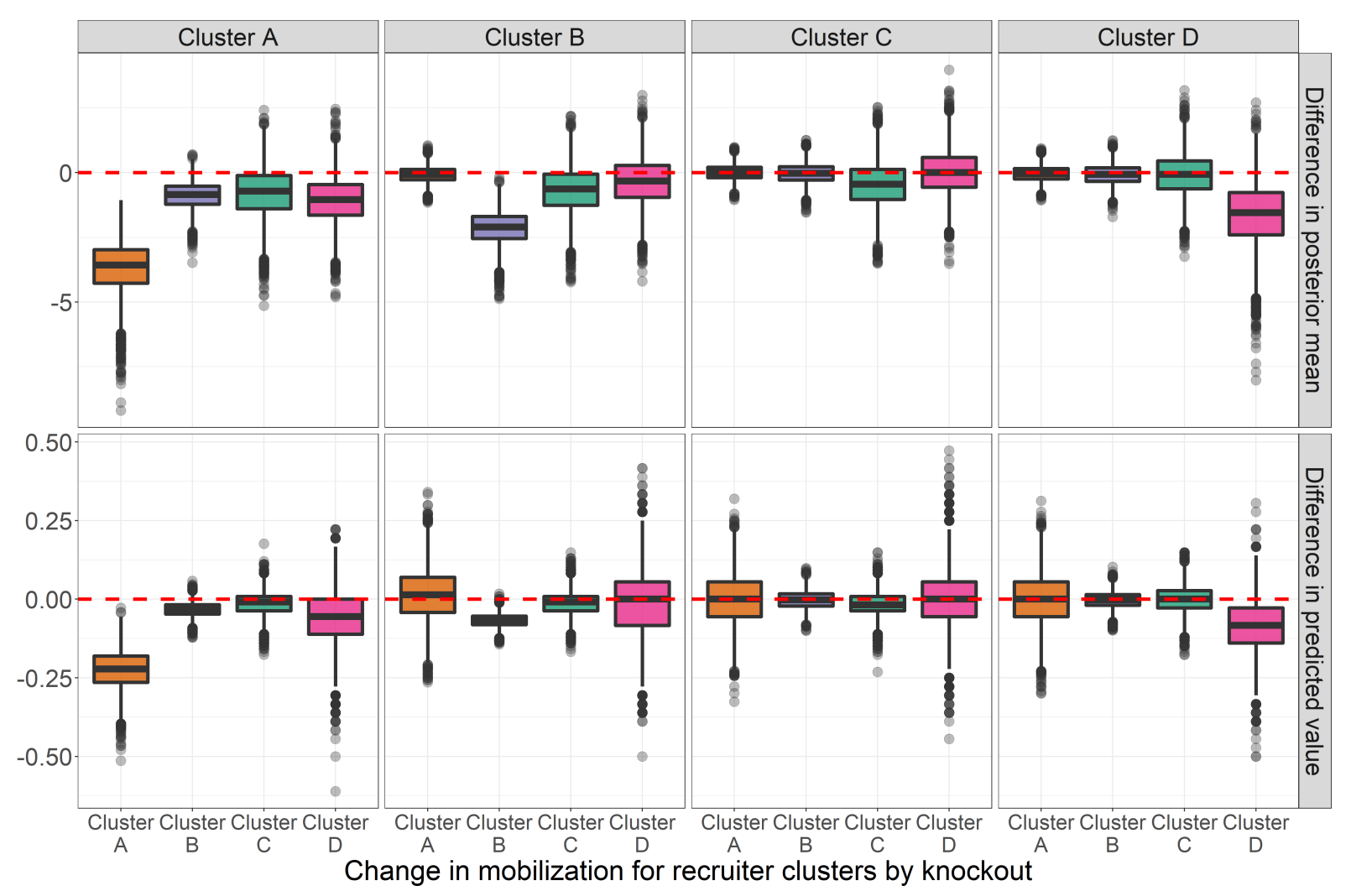

Figure 6: The difference in PR following simulated network knockouts. We see the changes in the difference in PR for each cluster with the columns corresponding to the knocked out cluster. All simulated knockouts decrease the remaining recruiters' PR, but the effect varies by cluster. 
exerts less damage than removing cluster A with a total of 4 recruiters when taking into account the sampling variability. To be fair, on average, recruiters in cluster A are much more active than recruiters in cluster B.

\subsection{Impact of Stress without Network Dependencies}

To assess the impact of network dependencies on network resilience, we use simulated data to compare knockout effects when there is no dependence in the data versus when there is dependence. Figure 7 presents the densities of the knockout effect based on 100 generated data. While the impacts of knockouts are different based on clusters, they are consistently smaller when there are dependencies in the data versus when there is no dependence. This result shows that Islamic recruitment is harder to destruct considering the dependencies in its network. Assuming independence in Islamic recruitment can lead to overoptimism and overestimation of the effectiveness of decapitation trikes.

\section{Conclusion}

In this paper, we demonstrate how latent space models can be used to help researchers understand network resilience. We apply our approach to demonstrate how terrorist organizations might be resilient in the face of external attacks. In so doing, this paper offers notable contributions to the extant research in political methods and terrorism. Before discussing the article's contributions, we wish to note two limitations. The first limitation relates to the data source. The analysis relies on Islamic State recruitment data. The journalist who gave us the data said that this was the full universe of documents; however, we may be missing some links within the Islamic State recruitment network. ${ }^{7}$ Although it is impossible to fully verify the Islamic State recruitment network data, we believe the network serves as a close approximation and a significant step forward in researchers' efforts to study mobilization and recruitment. Second, we analyze how static network structures are resilient to node knockouts. Future analyses can build on this work to explore how dynamic social network systems would adapt to network knockouts. And third, we explore the Islamic State recruitment network between the years 2010 to 2015. During this time period, the Islamic State was primarily fighting against the Iraqi and Syrian governments. However, in late 2014, the United

${ }^{7}$ One of the authors on this paper took steps to measure the representativeness of the data and was advised by the United States Federal Bureau of Investigations to stop their follow up efforts over safety concerns. 


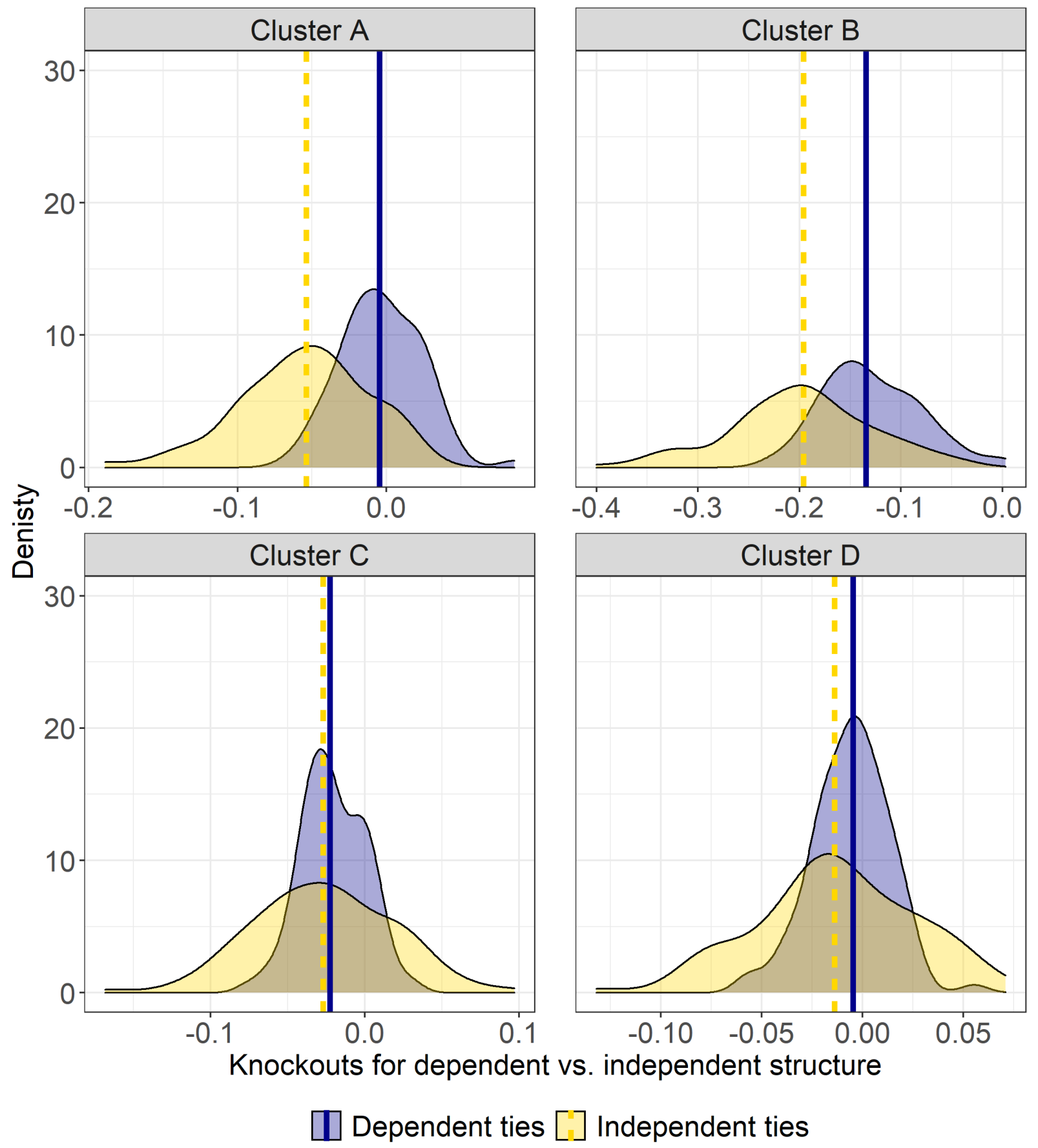

Figure 7: Simulated knockouts for independent (yellow) versus dependent (blue) recruitment network. The vertical lines indicate the median of the distribution of the knockout effect. Across all cluster knockouts, the dependent structure is more resilient to the external node knockouts. 
States and several other great powers entered the conflict against the Islamic State. In turn, the combatants during this analysis period could be different from combatants who decided to join the Islamic State after other states became engaged in the conflict, as fighting on behalf of the Islamic State likely became an even riskier endeavor.

The limitation notwithstanding, this paper advances our understanding of networks and terrorism. First, we offer a novel method for studying network resilience using latent space models. Second, we apply our novel methodological approach to an important case - understanding how foreign fighter mobilization for the Islamic State would be affected by the knockout of recruiters. Our research suggests that national security policies that seek to reduce Islamic State recruitment should consider the resilience of the Islamic recruitment network. The impact of external attacks tends to be minimized given network dependencies. Thus, coordinated knockouts of central terrorist recruiters may be more effective than widespread attacks against the system.

Together, these findings have important implications for research on network resilience as well as the United States and its allies' counterterrorism efforts. In particular, our proposed methodological approach and novel data demonstrate the conditions under which terrorist organizations can be efficiently disrupted through the removal of key actors. Through this approach, counterterrorism policies can be designed to efficiently demobilize new fighters, and future research should explore under what circumstances we can anticipate quick recovery and adaptation of the network as these issues are vitally important in governments' efforts to disrupt and dismantle terrorist organizations. 


\section{References}

Abdel-Jelil, Mohamed, Kartika Bhatia, Anne Brockmeyer, Quy-Toan Do and Clement Joubert. 2015. "Unemployment and violent extremism: Evidence from Daesh foreign recruits." World Bank: Policy Research Working Paper 13.

Ahmida, Ali Abdullatif. 1994. The making of modern Libya: state formation, colonization, and resistance, 1830-1932. SUNY Press.

Albert, R, H Jeong and AL Barabasi. 2000a. "Attack and error tolerance of com plex networks." Nature 406(6794):378-382.

Albert, Réka, Hawoong Jeong and Albert-László Barabási. 2000b. "Error and attack tolerance of complex networks." nature 406(6794):378-382.

Asal, Victor H, Gary A Ackerman and R Karl Rethemeyer. 2012. "Connections can be toxic: Terrorist organizational factors and the pursuit of CBRN weapons." Studies in Conflict 8 Terrorism $35(3): 229-254$.

Asal, Victor H, Hyun Hee Park, R Karl Rethemeyer and Gary Ackerman. 2016. "With friends like these... why terrorist organizations ally." International Public Management Journal 19(1):1-30.

Atran, Scott. 2010. "Pathways to and from violent extremism: The case for science-based field research." Statement before the Senate Armed Services Subcommittee on Emerging Threats 8 Capabilities 10.

Atran, Scott. 2011. Who becomes a terrorist today? In The ethics and efficacy of the global war on terrorism. Springer pp. $45-58$.

Ayling, Julie. 2009. "Criminal organizations and resilience." International Journal of Law, Crime and Justice 37(4):182-196.

Bacon, Tricia. 2018. Why terrorist groups form international alliances. University of Pennsylvania Press.

Baggio, Jacopo A, Shauna B BurnSilver, Alex Arenas, James S Magdanz, Gary P Kofinas and Manlio De Domenico. 2016. "Multiplex social ecological network analysis reveals how social 
changes affect community robustness more than resource depletion." Proceedings of the National Academy of Sciences 113(48):13708-13713.

Barnett, George A and Ke Jiang. 2016. "Resilience of the World Wide Web: a longitudinal two-mode network analysis." Social Network Analysis and Mining 6(1):1-15.

Bastiampillai, Tarun, Stephen Allison and Sherry Chan. 2013. "Is depression contagious? The importance of social networks and the implications of contagion theory." Australian $\& 5$ New Zealand Journal of Psychiatry 47(4):299-303.

Bessi, Alessandro, Fabio Petroni, Michela Del Vicario, Fabiana Zollo, Aris Anagnostopoulos, Antonio Scala, Guido Caldarelli and Walter Quattrociocchi. 2016. "Homophily and polarization in the age of misinformation." The European Physical Journal Special Topics 225(10):2047-2059.

bin Khaled Al-Saud, Abdullah. 2019. Saudi foreign fighters: Analysis of leaked Islamic State entry documents. Technical report King's College London: King Faisal Center for Research and Islamic Studies.

Bisgin, Halil, Hasan Arslan and Yusuf Korkmaz. 2019. Analyzing the dabiq magazine: The language and the propaganda structure of isis. In International Conference on Social Computing, Behavioral-Cultural Modeling and Prediction and Behavior Representation in Modeling and Simulation. Springer pp. 1-11.

Boutyline, Andrei and Robb Willer. 2017. "The social structure of political echo chambers: Variation in ideological homophily in online networks." Political psychology 38(3):551-569.

Broder, Andrei, Ravi Kumar, Farzin Maghoul, Prabhakar Raghavan, Sridhar Rajagopalan, Raymie Stata, Andrew Tomkins and Janet Wiener. 2011. Graph structure in the web. In The Structure and Dynamics of Networks. Princeton University Press pp. 183-194.

Callimachi, Rukmini. 2018. "The ISIS files: We unearthed thousands of internal documents that help explain how the Islamic State stayed in power so long." New York Times .

URL: $\quad$ https://www.nytimes.com/interactive/2018/04/04/world/middleeast/isis-documentsmosul-iraq.html

Carley, Kathleen M. 2006. "Destabilization of covert networks." Computational \& Mathematical Organization Theory 12(1):51-66. 
Carley, Kathleen M, Jeffrey Reminga and Natasha Kamneva. 2003. Destabilizing terrorist networks. In 8th International Command and Control Research and Technology Symposium, National Defense War College, Washington DC.

CBS News. 2019. "Docs appear to reveal info on dozens of ISIS recruits." CBS News Online . https://www.cbsnews.com/news/ isis-application-forms-documents-intelligence-haul-terror-group-recruits/.

Centola, Damon M. 2013. "Homophily, networks, and critical mass: Solving the start-up problem in large group collective action." Rationality and society 25(1):3-40.

Chatfield, Akemi Takeoka, Christopher G Reddick and Uuf Brajawidagda. 2015. Tweeting propaganda, radicalization and recruitment: Islamic state supporters multi-sided twitter networks. In proceedings of the 16th annual international conference on digital government research. pp. 239249.

Chen, Pin-Yu and Alfred O Hero. 2014. "Assessing and safeguarding network resilience to nodal attacks." IEEE Communications Magazine 52(11):138-143.

Choate, Mark I. 2007. "Identity politics and political perception in the European settlement of Tunisia: the French colony versus the Italian colony." French Colonial History 8(1):97-109.

Choi, Myunggoon, Yoonmo Sang and Han Woo Park. 2014. "Exploring political discussions by Korean Twitter users: A look at opinion leadership and homophily phenomenon." Aslib Journal of Information Management .

Cohen, Reuven, Keren Erez, Daniel Ben-Avraham and Shlomo Havlin. 2000. "Resilience of the internet to random breakdowns." Physical review letters 85(21):4626.

Cohen, Reuven, Keren Erez, Daniel Ben-Avraham and Shlomo Havlin. 2001. "Cohen, Erez, benAvraham, and Havlin Reply." Physical Review Letters 87(21):219802.

Colleoni, Elanor, Alessandro Rozza and Adam Arvidsson. 2014. "Echo chamber or public sphere? Predicting political orientation and measuring political homophily in Twitter using big data." Journal of communication 64(2):317-332. 
Dincelli, Ersin, Yuan Hong and Nic DePaula. 2016. "Information diffusion and opinion change during the gezi park protests: Homophily or social influence?" Proceedings of the Association for Information Science and Technology 53(1):1-5.

Dong, Gaogao, Jingfang Fan, Louis M Shekhtman, Saray Shai, Ruijin Du, Lixin Tian, Xiaosong Chen, H Eugene Stanley and Shlomo Havlin. 2018. "Resilience of networks with community structure behaves as if under an external field." Proceedings of the National Academy of Sciences 115(27):6911-6915.

Donohue, Ian, Helmut Hillebrand, José M Montoya, Owen L Petchey, Stuart L Pimm, Mike S Fowler, Kevin Healy, Andrew L Jackson, Miguel Lurgi, Deirdre McClean et al. 2016. "Navigating the complexity of ecological stability." Ecology letters 19(9):1172-1185.

Dorff, Cassy, Max Gallop and Shahryar Minhas. 2020. "Networks of violence: Predicting conflict in Nigeria." The Journal of Politics 82(2):476-493.

Dunne, Jennifer A, Richard J Williams and Neo D Martinez. 2002. "Food-web structure and network theory: the role of connectance and size." Proceedings of the National Academy of Sciences 99(20):12917-12922.

Edgerton, Jared. 2022. "Suicide bomber mobilization and kin and peer ties." Social Networks $70: 36-54$.

Eom, Young-Ho. 2018. "Resilience of networks to environmental stress: From regular to random networks." Physical Review E 97(4):042313.

Fernández-Martínez, Elena, Elena Andina-Díaz, Rosario Fernández-Peña, Rosa García-López, Iván Fulgueiras-Carril and Cristina Liébana-Presa. 2017. "Social networks, engagement and resilience in university students." International journal of environmental research and public health $14(12): 1488$.

Friel, Nial, Riccardo Rastelli, Jason Wyse and Adrian E Raftery. 2016. "Interlocking directorates in Irish companies using a latent space model for bipartite networks." Proceedings of the National Academy of Sciences 113(24):6629-6634. 
Gade, Emily Kalah, Michael Gabbay, Mohammed M Hafez and Zane Kelly. 2019. "Networks of cooperation: Rebel alliances in fragmented civil wars." Journal of Conflict Resolution 63(9):20712097.

Gallop, Max and Shahryar Minhas. 2021. "A network approach to measuring state preferences." Network Science 9(2):135-152.

Gallos, Lazaros K, Reuven Cohen, Panos Argyrakis, Armin Bunde and Shlomo Havlin. 2005. "Stability and topology of scale-free networks under attack and defense strategies." Physical review letters 94(18):188701.

Gao, Jianxi, Baruch Barzel and Albert-László Barabási. 2016. "Universal resilience patterns in complex networks." Nature 530(7590):307-312.

Griffith, Daniel A and Yongwan Chun. 2015. "Spatial autocorrelation in spatial interactions models: geographic scale and resolution implications for network resilience and vulnerability." Networks and Spatial Economics 15(2):337-365.

Hafez, Mohammed M. 2016. "The ties that bind: How terrorists exploit family bonds." CTC Sentinel 9(2):15-18.

Handcock, Mark S, Adrian E Raftery and Jeremy M Tantrum. 2007. "Model-based clustering for social networks." Journal of the Royal Statistical Society: Series A (Statistics in Society) 170(2):301-354.

Heider, Fritz. 1944. "Social perception and phenomenal causality." Psychological review 51(6):358.

Heider, Fritz. 1946. "Attitudes and cognitive organization." The Journal of psychology 21(1):107112.

Hellsten, Sirkku. 2016. Radicalisation and terrorist recruitment among Kenya's youth. Nordiska Afrikainstitutet.

Hoff, Peter. 2008. Modeling homophily and stochastic equivalence in symmetric relational data. In Advances in neural information processing systems. pp. 657-664.

Hoff, Peter D. 2005. "Bilinear mixed-effects models for dyadic data." Journal of the american Statistical association 100(469):286-295. 
Hoff, Peter D, Adrian E Raftery and Mark S Handcock. 2002. "Latent space approaches to social network analysis." Journal of the american Statistical association 97(460):1090-1098.

Horowitz, Michael C and Philip BK Potter. 2014. "Allying to kill: Terrorist intergroup cooperation and the consequences for lethality." Journal of Conflict Resolution 58(2):199-225.

Huber, Gregory A and Neil Malhotra. 2017. "Political homophily in social relationships: Evidence from online dating behavior." The Journal of Politics 79(1):269-283.

Janssen, Marco A, Örjan Bodin, John M Anderies, Thomas Elmqvist, Henrik Ernstson, Ryan RJ McAllister, Per Olsson and Paul Ryan. 2006. "Toward a network perspective of the study of resilience in social-ecological systems." Ecology and Society 11(1).

Jeong, Hawoong, Sean P Mason, A-L Barabási and Zoltan N Oltvai. 2001. "Lethality and centrality in protein networks." Nature 411(6833):41-42.

Johnston, Patrick B. 2012. "Does decapitation work? Assessing the effectiveness of leadership targeting in counterinsurgency campaigns." International Security 36(4):47-79.

Johnston, Patrick B, Jacob N Shapiro, Howard J Shatz, Benjamin Bahney, Danielle F Jung, Patrick K Ryan and Jonathan Wallace. 2016. Foundations of the Islamic State: management, money, and terror in Iraq, 2005-2010. Rand Corporation.

Jordan, Jenna. 2009. "When heads roll: Assessing the effectiveness of leadership decapitation." Security Studies 18(4):719-755.

Kong, Feng, Xu Wang, Siyuan Hu and Jia Liu. 2015. "Neural correlates of psychological resilience and their relation to life satisfaction in a sample of healthy young adults." Neuroimage 123:165172.

Krebs, Valdis E. 2002. "Mapping networks of terrorist cells." Connections 24(3):43-52.

Krivitsky, Pavel N, Mark S Handcock, Adrian E Raftery and Peter D Hoff. 2009. "Representing degree distributions, clustering, and homophily in social networks with latent cluster random effects models." Social networks 31(3):204-213.

LaFree, Gary and Laura Dugan. 2007. "Introducing the global terrorism database." Terrorism and political violence 19(2):181-204. 
Laishram, Ricky, Ahmet Erdem Sariyüce, Tina Eliassi-Rad, Ali Pinar and Sucheta Soundarajan. 2018. Measuring and improving the core resilience of networks. In Proceedings of the 2018 World Wide Web Conference. pp. 609-618.

Lakomy, Miron. 2021. "Recruitment and incitement to violence in the Islamic State's online propaganda: Comparative analysis of Dabiq and Rumiyah." Studies in Conflict $\mathscr{G}$ Terrorism 44(7):565580.

Larson, Jennifer M. 2021. "Networks of Conflict and Cooperation." Annual Review of Political Science 24:89-107.

Larson, Jennifer M and Janet I Lewis. 2017. "Ethnic networks." American Journal of Political Science 61(2):350-364.

Larson, Jennifer M and Janet I Lewis. 2018. "Rumors, kinship networks, and rebel group formation." International Organization 72(4):871-903.

Lee, Wesley, Tyler H McCormick, Joshua Neil and Cole Sodja. 2019. "Anomaly detection in large scale networks with latent space models." arXiv preprint arXiv:1911.05522 .

Lindelauf, Roy, Peter Borm and Herbert Hamers. 2011. Understanding terrorist network topologies and their resilience against disruption. In Counterterrorism and Open Source Intelligence. Springer pp. 61-72.

Liu, Xindong, Mohammad Shahidehpour, Zuyi Li, Xuan Liu, Yijia Cao and Zhaohong Bie. 2016. "Microgrids for enhancing the power grid resilience in extreme conditions." IEEE Transactions on Smart Grid 8(2):589-597.

Magouirk, Justin, Scott Atran and Marc Sageman. 2008. "Connecting terrorist networks." Studies in Conflict $\mathcal{E}$ Terrorism 31(1):1-16.

Malet, David. 2013. Foreign fighters: Transnational identity in civil conflicts. Oxford University Press.

Malm, Aili, Rebecca Nash and Ramin Moghadam. 2017. "Social network analysis and terrorism." The Handbook of the Criminology of Terrorism pp. 221-231. 
Mannes, Aaron. 2008. "Testing the snake head strategy: Does killing or capturing its leaders reduce a terrorist group's activity?" The Journal of International Policy Solutions 9.

Maoz, Zeev. 2012. "Preferential attachment, homophily, and the structure of international networks, 1816-2003." Conflict Management and Peace Science 29(3):341-369.

Matthew, Richard and George Shambaugh. 2005. "The limits of terrorism: a network perspective." International Studies Review 7(4):617-627.

May, Robert M. 2006. "Network structure and the biology of populations." Trends in Ecology 83 Evolution 21(7):394-399.

McPherson, Miller, Lynn Smith-Lovin and James M Cook. 2001. "Birds of a feather: Homophily in social networks." Annual review of sociology 27(1):415-444.

Medina, Richard M. 2014. "Social network analysis: a case study of the Islamist terrorist network." Security Journal 27(1):97-121.

Memon, Nasrullah, Henrik Legind Larsen, David L Hicks and Nicholas Harkiolakis. 2008. Retracted: detecting hidden hierarchy in terrorist networks: some case studies. In International Conference on Intelligence and Security Informatics. Springer pp. 477-489.

Milton, Daniel and Bryan Price. 2020. "Too central to fail? Terror networks and leadership decapitation." International Interactions pp. 1-25.

Minhas, Shahryar, Peter D Hoff and Michael D Ward. 2019. "Inferential approaches for network analysis: Amen for latent factor models." Political Analysis 27(2):208-222.

Moon, Il-Chul and Kathleen M Carley. 2007. "Modeling and simulating terrorist networks in social and geospatial dimensions." IEEE Intelligent Systems 22(5):40-49.

Morris, Andrea Michelle. 2020. "Who Wants to Be a Suicide Bomber? Evidence from Islamic State Recruits." International Studies Quarterly 64(2):306-315.

Newman, Lenore and Ann Dale. 2005. "Network structure, diversity, and proactive resilience building: a response to Tompkins and Adger." Ecology and society 10(1).

Newman, Mark EJ. 2003. "Mixing patterns in networks." Physical Review E 67(2):026126. 
Nielsen, Richard A. 2017. Deadly clerics: Blocked ambition and the paths to jihad. Cambridge University Press.

Norman, Robert Z et al. 1965. "Structural models: An introduction to the theory of directed graphs.".

O’Neale, Dion RJ et al. 2019. "Latent space generative model for bipartite networks." arXiv preprint arXiv:1910.12488.

Opper, Sonja, Victor Nee and Stefan Brehm. 2015. "Homophily in the career mobility of China's political elite." Social Science Research 54:332-352.

Orozobekova, Almakan. 2016. "The Mobilization and Recruitment of Foreign Fighters: The Case of Islamic State, 2012-2014." Connections 15(3):83-100.

Phan, Tuan Q and Edoardo M Airoldi. 2015. "A natural experiment of social network formation and dynamics." Proceedings of the National Academy of Sciences 112(21):6595-6600.

Phillips, Brian J. 2019. "Terrorist group rivalries and alliances: testing competing explanations." Studies in Conflict \& Terrorism 42(11):997-1019.

Price, Bryan C. 2012. "Targeting top terrorists: How leadership decapitation contributes to counterterrorism." International Security 36(4):9-46.

Reynolds, Sean C and Mohammed M Hafez. 2019. "Social network analysis of German foreign fighters in Syria and Iraq." Terrorism and Political Violence 31(4):661-686.

Ryckman, Kirssa Cline. 2020. "Lasting peace or temporary calm? Rebel group decapitation and civil war outcomes." Conflict Management and Peace Science 37(2):172-192.

Salter-Townshend, Michael and Tyler H McCormick. 2017. "Latent space models for multiview network data." The annals of applied statistics 11(3):1217.

Sarkar, Purnamrita and Andrew W Moore. 2005. "Dynamic social network analysis using latent space models." Acm Sigkdd Explorations Newsletter 7(2):31-40.

Sarkar, Purnamrita and Andrew W Moore. 2006. Dynamic social network analysis using latent space models. In Advances in Neural Information Processing Systems. pp. 1145-1152. 
Sarkar, Purnamrita, Sajid M Siddiqi and Geogrey J Gordon. 2007. A latent space approach to dynamic embedding of co-occurrence data. In Artificial Intelligence and Statistics. PMLR pp. 420427.

Scheffer, Marten, J Elizabeth Bolhuis, Denny Borsboom, Timothy G Buchman, Sanne MW Gijzel, Dave Goulson, Jan E Kammenga, Bas Kemp, Ingrid A van de Leemput, Simon Levin et al. 2018. "Quantifying resilience of humans and other animals." Proceedings of the National Academy of Sciences 115(47):11883-11890.

Schneider, Christian M, André A Moreira, José S Andrade, Shlomo Havlin and Hans J Herrmann. 2011. "Mitigation of malicious attacks on networks." Proceedings of the National Academy of Sciences 108(10):3838-3841.

Sewell, Daniel K and Yuguo Chen. 2015. "Latent space models for dynamic networks." Journal of the American Statistical Association 110(512):1646-1657.

Speckhard, Anne, Molly Ellenberg, Haider Shaghati and Neima Izadi. 2020. "Hypertargeting facebook profiles vulnerable to ISIS recruitment with" Breaking the ISIS brand counter narrative video clips" in multiple facebook campaigns." Journal of Human Security 16(1):16-29.

Sterman, David. June 2018. The Islamic State's veterans: Contrasting the cohorts with jihadi experience in Libya and Afghanistan. Technical report West Point: Combating Terrorism Center.

Wang, Selena. 2021. "Recent Integrations of Latent Variable Network Modeling With Psychometric Models." Frontiers in psychology 12:773289-773289.

Wang, Selena Shuo, Subhadeep Paul and Paul De Boeck. 2021. "Joint Latent Space Model for Social Networks with Multivariate Attributes." arXiv preprint arXiv:1910.12128v2.

Wasserman, Stanley and Dawn Iacobucci. 1991. "Statistical modelling of one-mode and two-mode networks: Simultaneous analysis of graphs and bipartite graphs." British Journal of Mathematical and Statistical Psychology 44(1):13-43.

Wasserman, Stanley and Katherine Faust. 1994. Social network analysis: Methods and applications. Vol. 8 Cambridge university press. 
Weare, Christopher, Juliet Musso and Kyu-Nahm Jun. 2009. "Cross-talk: The role of homophily and elite bias in civic associations." Social Forces 88(1):147-173.

Weimann, Gabriel. 2016. The emerging role of social media in the recruitment of foreign fighters. In Foreign fighters under international law and beyond. Springer pp. 77-95.

Wuchty, Stefan. 2014. "Controllability in protein interaction networks." Proceedings of the National Academy of Sciences 111(19):7156-7160.

Yaoren, Kenneth Yeo. 2019. "Leadership Decapitation and the Impact on Terrorist Groups." Counter Terrorist Trends and Analyses 11(3):7-12.

Yoo, Suhyung and Hwasoo Yeo. 2016. "Evaluation of the resilience of air transportation network with adaptive capacity." International Journal of Urban Sciences 20(sup1):38-49.

Zhang, Limiao, Guanwen Zeng, Daqing Li, Hai-Jun Huang, H Eugene Stanley and Shlomo Havlin. 2019. "Scale-free resilience of real traffic jams." Proceedings of the National Academy of Sciences 116(18):8673-8678.

Zitnik, Marinka, Marcus W Feldman, Jure Leskovec et al. 2019. "Evolution of resilience in protein interactomes across the tree of life." Proceedings of the National Academy of Sciences 116(10):44264433. 